Magnus Ronn | LM Tucker | Ron Dulaney Jr. Sinclair, Mousazadeh, Safarzadeh | James Shraiky Marci Uihlein | Tang, Anderson, Aksamija, Hodge

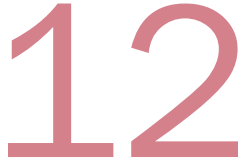

volume 9 | issue 1

\section{Sensory-Based Design \& Epilepsy: Analyzing effects of design innovations on patient treatment and recovery}

\author{
James Shraiky, Arizona State University \\ Research \\ Joshua Schoonover (ASU), Joseph Sirven (Mayo Clinic in \\ Arizona), Luann Helepololei (Mayo Clinic in Arizona)
}

\begin{abstract}
The patient room has more influence on neurological, physiological and biological responses than any other area within a healthcare environment. When it comes to the treatment of epilepsy, the patient room often acts as a refuge from and instigator of seizure activity, depending on patient condition or status. Inspired by this dichotomy, this research report explores specific design parameters that affect the spectrum of epileptic treatment, from instigation to recovery. At its core, this research identifies specific environmental elements that can assist in instigating an episode and recovery post seizure.
\end{abstract}

Inclusions: Patients between the ages of 18 and 65 who are admitted for monitoring purposes only.

Exclusions: Post-surgical procedure patients. Patients who are admitted for any type of pre-surgical procedure. Patients who are admitted less than two days.

Limitations: The Epilepsy Monitoring Unit was designed as a Medical Surgical Unit.

\section{Introduction}

Epilepsy is one of the most common neurological disorders in the U.S., amounting to more than $\$ 15$ billion in direct and indirect costs and affecting more people than Parkinson's disease, multiple sclerosis and Lou Gehrig's disease combined (Baruchin, 2007; Epilepsy Foundation, n.d.). The statistics associated with epilepsy and seizures are fairly jarring for a neurological disorder that few people outside of the neuroscience community are willing to actively discuss or support - a disorder still handcuffed to a senseless social stigma. In her New York Times article on the social consequences of epilepsy, Baruchin (2007) remarked that the centuries-old stigma associated with epilepsy, to this day, "hampers care, public recognition and the ability to raise money for research" (para. 5). If research efforts within the neuroscience field are hobbled due to a lack of financial and public support, it should not come as a surprise that epilepsy-related research in disciplines relating to space design are largely non-existent. This article hopes to take a small step toward filling that void by investigating the role of physical design interventions in enhancing epilepsy diagnosis and treatment.

It is not a surprise to say that the successful treatment and management of epilepsy depends first on an accurate diagnosis. Some patients demonstrate classic, telltale signs of epilepsy and are diagnosed accordingly, while other seemingly obvious seizure activity may actually be the result of non-epilepsy related psychological responses. Given the broad range of symptoms, causes, triggers, severity, seizure types (including non-epileptic seizures), and so on, diagnosing epilepsy is often anything but a simple $A+B=C$ proposition. Enter the epilepsy monitoring unit (EMU), an inpatient unit equipped with the medical expertise, staff and technology necessary to observe, analyze, diagnose and treat seizure activity in patients. And, it is the EMU - more specifically, the patient room within an EMU - that serves as the setting for this particular research endeavor.

Unlike any other area in a healthcare facility, the patient room has the ability to influence a patient's neurological, physiological and biological responses. This is especially true of a patient room in an EMU, in which the stimulation of these responses is one of the primary objectives. Patients experiencing worsening or unusual seizure-like episodes are voluntarily admitted into the EMU, knowing that they will be put through a series of tests and experiences aimed toward stressing and over-stimulating 


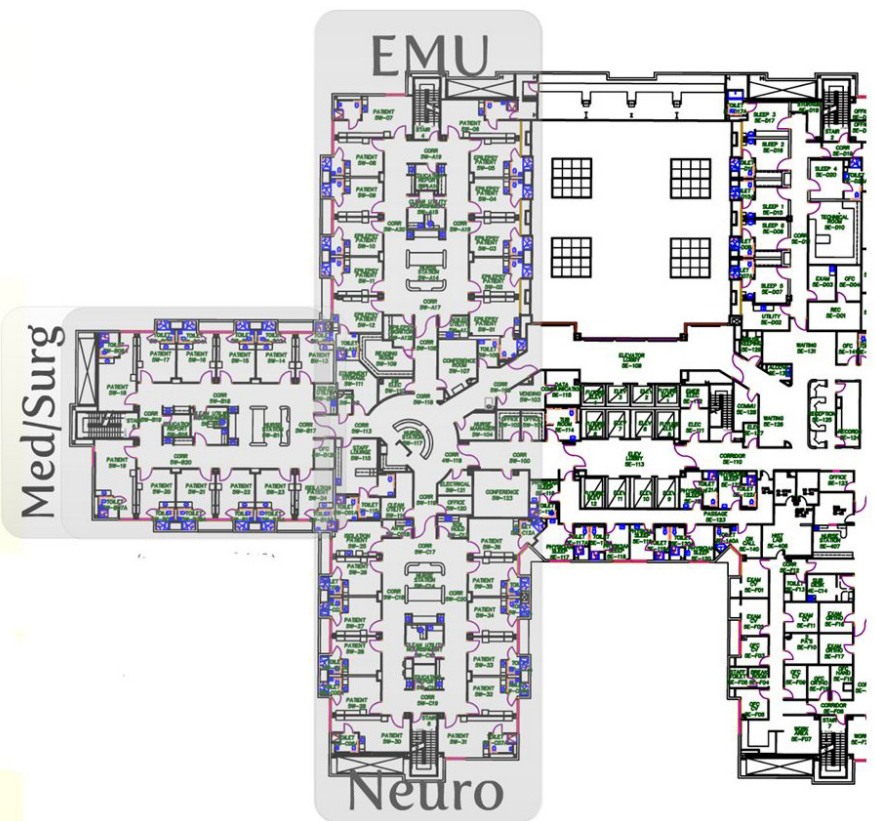

Image 1: Mayo Clinic EMU Floor Plan

the senses, as well as physically and psychologically exhausting them to the point of seizure, all while their every move is audibly and visually monitored 24 hours a day.

The length of a patient's stay in an EMU, which averages approximately five days, is predicated on the staff's ability to gather enough data from intentionally instigated seizure episodes in order to analyze and develop an accurate diagnosis and treatment plan. Strobe lights, exercise bikes, sleep deprivation and other measures are employed in an attempt to stimulate or stress the patient to the point of seizure. The sooner a seizure is triggered, the sooner data collection begins, and ultimately, the better the patient outcome.

For the last 30 years, research has suggested that the design of the built environment of healthcare facilities impacts patient outcomes (Ulrich et al., 2008). With the understanding that sensory-based and stressrelated elements are widely used in the instigation of seizure activity, it was the hypothesis of those involved in this study that opportunities exist within the EMU for the incorporation of various sensory-based design interventions in optimizing the processes utilized in the instigation of seizure activity in patients. This study set out to explore the potential of and serve as a basis for employing design interventions that channel sensory and stress-inducing elements for more efficient and effective means of instigating seizure activity. Furthermore, due to the intense nature of an epileptic episode, this study also looks to identify design interventions that aid the recover process post seizure.
Description of the EMU at Mayo Clinic Hospital in Phoenix, Arizona Despite the highly-unique services and activities that take place within an EMU, the EMU at the Mayo Clinic Hospital in Phoenix, Arizona, is quite similar, in terms of floor plan, spatial dimensions and physical layout, as other units within the facility. The unit houses a total of 12 patient beds: six are designated for epilepsy monitoring and the other six are dedicated to medical/surgical admissions. When the six beds are not being used for epilepsy monitoring, they flex for medical/surgical inpatient admissions. (see image 1)

The EMU at Mayo is a Level IV epilepsy center, meaning it handles all cases. Each year, approximately 250 to 280 patients, both domestic and international, are voluntarily admitted into the unit. The length of a patient's stay can range from two days to 30 days, with the average length of stay being five days. Every moment of a patient's stay is monitored via video and audio technology, as well as personal observation. In addition to the patient rooms, there is a surveillance room located in the unit, where at least one member of the staff is constantly surveying a bank of six monitors playing audio and visual feeds from each patient room. Should unusual or seizure-related activity be detected, an alarm is sounded and staff members immediately react to the patient requiring medical attention.

As previously mentioned, staff members within the unit employ a variety of techniques to induce seizure activity in patients, including physical stress and exhaustion, sleep deprivation, medication deprivation, photostimulation, and environmental stressors. The majority of these seizureinstigating activities occur within the patient's room. When seizure events are successfully triggered, the data is collected and used for diagnosis and treatment purposes.

For the majority of their stay, patients are restricted, quite literally, to their beds. In order to keep the patients within a visual plane of the video cameras and staff, and to prevent injury from falling, patients are physically strapped in their beds using a restrictive belt.

Once sufficient data is collected to make a diagnosis and treatment strategy, patients' medication regimens resume, and once stable, they are discharged from the unit.

\section{Justification}

Epilepsy carries a significant social burden for both patients and families affected by the condition. According to a study by Ellis and colleagues, families with children who suffer from epilepsy are more likely to have weaker family bonds than families with children who suffer from other chronic illness (Ellis and colleagues, 2000). This dynamic is found to be less supportive, affectionate and performance-oriented than their counterparts (Baker et al., 2005). Similarly, individuals, particularly young 
adults, living with epilepsy often report feeling stigmatized by the disease (The Centers for Disease Control [CDC], 1997). Managing the disease in the public realm often interferes with basic developmental functions, such as work, social interaction and personal care - all of which help create a sense of identity. The stigma of epilepsy alone is a major cause for reducing the quality of life for individuals suffering from the disease (Baker, et. al, 1997) So much so, that the Center for Disease Control's 1997 Living Well with Epilepsy Conference suggested that combating the stigma should be a high national priority (CDC). Additionally, and based on literature review, there is a big gap in research on how the physical design of the EMU may contribute to better patient outcomes.

\section{Methodology}

As previously stated, the goal of this study is to explore specific sensorybased, stress-inducing design elements that can be integrated into the EMU patient room environment as a means of effectively and efficiently instigating seizure activity and assisting in the recovery process post seizure. A post occupancy evaluation was utilized for the first phase of the study. Researchers created a post-descriptive survey and administered it to a convenience sample of clinical staff and patients at the Phoenix Mayo Clinic's Epilepsy Monitoring Unit. While investigating data collection instruments and after a comprehensive review of current literature, researchers discovered that there were no standardized questionnaires currently available to assess the goals of interest for this study. Therefore, the investigators developed surveys to identify, from patients and staff perspectives, elements of the built environment that cause stress prior to seizure activities and those that assist in the recovery process post seizure. Survey questions were based on the outcome of a series of interviews and focus groups with the EMU clinical staff, during which, they viewed multiple videos of patient experiences while at the EMU. The videos presented patient experience prior, during, and post seizure activities. Researchers then created two different surveys from the interview and focus groups - one targeted to capture the patient experience and the other formulated to capture the staff's perception of the patient experience.

\section{Survey Results}

Following the development of the patient survey, 22 EMU patients completed 17 short-answer and multiple-choice questions regarding the patient room design, stress and recovery points and their overall experience while in the EMU. The response scale ranged from 1 to 5 (1 = Strongly agree; 2 = Somewhat agree; 3 = Unsure; 4 = Somewhat disagree; 5 = Strongly disagree). Participants could also select "not applicable" as a response. In addition to inquiring about the room design and delivery of treatment, the researchers also wanted to know whether patients could identify specific design elements that caused stress or assisted in recovery. The survey questions were followed by a comment section asking for specific design-based instigators and elements of recovery. Similar to the patient survey format, 37 clinical staff completed the staff survey with questions regarding the patient room design and the stress-inducing and/or recovery attributes of the room.

\section{Data Analysis}

After analyzing both sets of survey responses, researchers found the data obtained was inconclusive. It informed the research team that the surveys were not effectively designed for the patients and staff to provide the design-based answers they desired for the study. What they gathered were patient and staff opinions, mostly satisfactory, on the delivery of care at the EMU. In some ways, the questions even confused the survey takers. Survey results did not clearly state whether the design of the patient room contributed to instigating seizure activities. In fact, 20 of the 22 patients responded unsure to questions regarding the matter. No specific design elements were identified for the instigation of seizure activity in the comment section. Patient feedback centered on clinical, non-design interventions like strobe lighting, exhaustion bike and sleep deprivation. Conversely, some patients indicated that the design of the room did contribute to an effective recovery process. Patients with views to the outdoors expressed how natural lighting and views of nature aided their recovery. Overall, for post seizure experiences, most patients felt very comfortable in the room and reported that it was well-suited for their needs.

Staff survey results were equally not as comprehensive as desired for the research goals. During attempts to instigate seizure activity in patients, most of the staff expressed beliefs that the design of the patient room was inadequate for both staff and patients because the size of the room does not allow for the storage of the necessary equipment. Beyond larger rooms and adequate storage space, no other design interventions were identified for causing seizures. When assessing the patient room during recovery, the majority of the staff also reported that patient rooms in the EMU aided treatment. Staff also referred to natural lighting and views from windows as supporting recovery post seizure.

Reevaluation of Methodology

After analyzing and synthesizing the survey responses, it became evident that a reevaluation of the research approach was necessary. As a result of this reevaluation, it was determined that the initial approach, conducting post occupancy evaluations, was not appropriate for achieving the intended research mission and goals. The surveys text format did not provide sufficient information related to specific design elements or environmental factors that may or may not impact activities or events related to the instigation of seizure activity.

Despite the unique spatial, technological and logistical needs of a patient room in an EMU, the rooms at Mayo Clinic's EMU are modeled after the facility's medical/surgical rooms. In other words, the EMU was retrofitted within an existing space, as opposed to the space being 
designed to serve the specialized needs, demands, and priorities of the EMU - causing the physical layout and design of the patient rooms appear inadequate. In order to better serve user needs in the EMU, it was hypothesized that the research needed to go beyond the boundaries of the post descriptive survey and "typical" patient room environment to identify the optimal design solutions for the highly-specialized EMU services and experiences.

Through another round of clinical staff interviews, it was discovered that unlike "typical" medical/surgical patients, EMU patients are voluntarily admitted. Their path to recovery requires an environment that triggers seizure activities and then supports them post seizure. Patient recovery and desired outcomes are predicated on the ability of EMU staff to instigate seizures so that the appropriate data can be collected and accurate diagnosis and treatment can be achieved.

After recognizing that the initial method was not a suitable approach, the next logical step was to propose a new research methodology. It was concluded, through patient and staff interviews, that identifying how to improve the EMU experience was to bring triggers and healing elements from "outside" life experiences into the patient room design. Through further research, the research team selected a new method of data collection, Experience-Based Design (EBD), because of how it maps the user experience within well-defined tools and helps researchers translate those experiences into design decision-making.

\section{Revised Methodology - Experienced-Based Design}

Experience-Based Design, an instrument used in collecting and measuring data in order to improve upon the delivery of general healthcare services, was developed for and in partnership with the United Kingdom's National Health Service (NHS) Institute for Innovation and Improvement. This approach is categorized by a collaborative design effort involving patients, care providers, visitors and other stakeholders associated with the healthcare experience. The premise is that documenting and understanding the "journey" of all healthcare users, as told by the individual, can provide valuable information for improving design, service and processes. Results reported by the NHS have been highly positive, "delivering the sort of care pathways that leave patients feeling safer, happier and more valued, and making staff feel more positive, rewarded and empowered" (NHS Institute for Innovation and Improvement, 2010).

There are four distinct phases to the EBD methodology: capturing the experience; understanding the experience; improving the experience; and, measuring the improvement. What follows is an explanation of each EBD phase as it relates to this investigation of the EMU.

\section{EBD Phase I - Capture the Experience}

Methodology Definition

This is an observation-based exercise that allows users to tell the story in their own words. Information is gathered through discovery interviews with open-ended questions, resulting in descriptive and explanatory answers. Experiential responses and recognition of emotion are core principals of EBD. To be as comprehensive as possible, it is essential to capture multiple perspectives from all user demographics (The EBD Approach Guide and Tools Book, pp. 18-21).

\section{EBD Implementation}

As previously mentioned, the goal for this revised methodology was to identify external elements that could be incorporated into the design of the EMU to both instigate seizure and support recovery. For this phase, investigators trained the EMU staff to conduct and document oral surveys/interviews of the patients. Oral survey questions were divided into two categories - pre and post seizure experiences inside the EMU and pre and post seizure experiences outside of the EMU in their daily lives.

The oral survey structure was organized into four segments. First, was gathering of patient medical background, which sought to obtain demographics and medical history. Second, was collecting patient hospital experiential data that captured the patients' status and experiences during their stay at EMU. Next, lifestyle data examined the patients' outside life experiences that were potentially linked to seizure triggers and recovery methods. Lastly, the patient room data identified existing design features within the EMU that triggered episodes as well as helped in post seizure recovery. Questions were descriptive and open-ended and patients were permitted to provide multiple answers.

For the patient room experience, being that they are already in an intense sensory environment, investigators discovered that EMU patients responded more in-depth to visual cues. Therefore, a 360 degree image of the existing patient room accompanied the oral the survey. (see image 2).

\section{EBD Phase II - Understand the Experience}

\section{Methodology Definition}

Once the user narratives are collected, the next step is to code answers and identify personal connections to the experiences. Personal connections can be both positive and negative and it is important to recognize and address both. When analyzing the user narratives, researchers classify touchpoints, i.e. words or signs that were associated with various parts of an experience. Touchpoints are important to each user and specific to his or her experience. They can be physical or nonphysical, emotional and sensory-based. Identifying touchpoints will provide insight on ways and means to improve upon an experience (The EBD Approach Guide and Tools Book, pp. 26-33). 


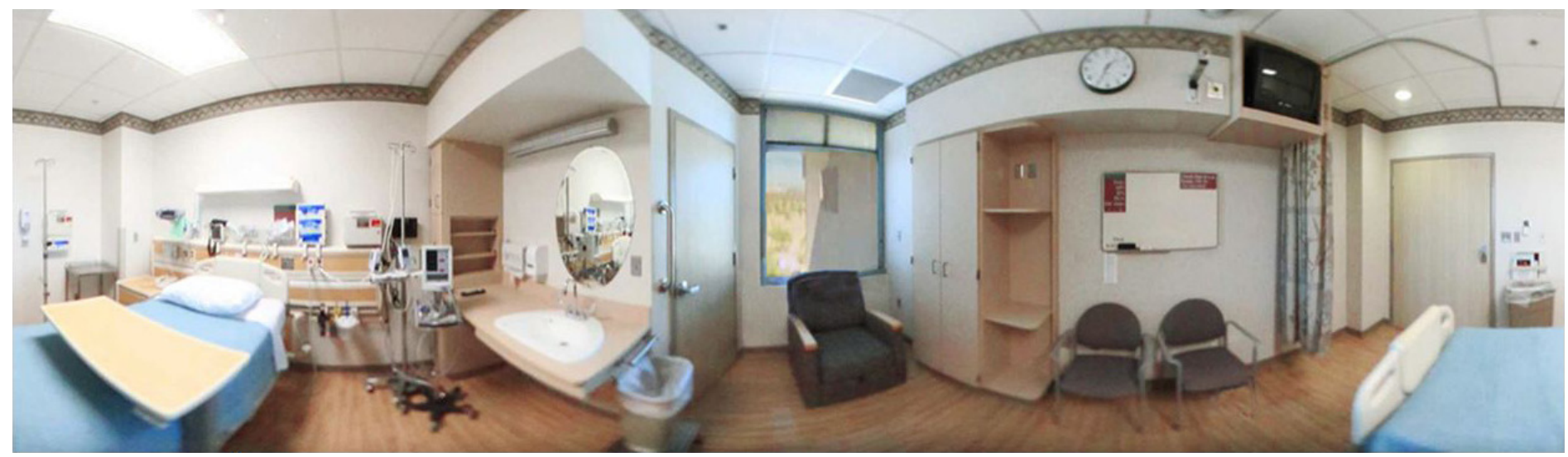

Image 2: $360^{\circ}$ Image of Existing EMU Patient Room

EBD Implementation

Survey answers were transcribed and condensed into the following themes and touch points.

\section{Outside the EMU}

\section{Levels of Seizure}

The acuity level of the disorder with our patient demographic ranged from mild to extreme. As such, the severity of the seizure activity also varied from mild, such as tension in the arm, to full body convulsions.

\section{Types of Trigger:}

The surveys concluded that outside triggers are unique to each patient. A majority reported that sensory-based elements triggered seizure activity. Nine patients listed distinct smells like burning rubber and cigarette smoke as instigators, four listed visual cues like family member photos and another four mentioned noises associated with traffic and screeching metal. Seizures were also linked environmental conditions, the participation in or recollection of social events and interaction with specific individuals. Four patients reported that they experience more acute and frequent episodes during seasonal temperature changes. Eight patients revealed that participating in or the memory of holiday events and other family gatherings triggers seizure activities while two people indicated that contact with ex family members, such as a former wife and mother in-law, instigated seizures.

\section{Types of Recovery Methods}

Techniques for patients recovering from seizures that occurred during their everyday lives included consuming media for entertainment, leisure activities and spiritual practices. For media, six patients prefer reading, seven work on the computer, 13 watch television, 16 listen to music and seven play video games. The leisure responses included 12 patients who spend time with family, five go on vacation, 12 enjoy nature and eight seek rest and relaxation. Spiritual practices involved prayer and meditation, with 11 patients participating in the activities.

\section{Inside the EMU}

Types of Triggers

Survey results suggest that there were no specific design interventions in the EMU that caused seizures. Common instigators included sleep deprivation, strobe lighting, medication level modification, exhaustion and hyperventilation.

Types of Recovery Method Investigators learned that recovery inside the patient room was similar to patients' lives outside of the EMU in that it involves, resting, relaxing, praying and meditating, reading, watching TV and movies, and spending time with loved ones. When coding all survey results, no matter how unique to the individual or typical to the patient demographic, we discovered a common denominator. With the exception of the manipulated medication levels, all of the experiences were sensory-based. To validate this observation, we referred back to the original POE staff focus group data and conducted additional interviews, where we identified more sensorybased experiences within their stories.

\section{EBD Phase III - Improve the Experience}

\section{Methodology Definition}

Once experiences are coded, the next step is to implement a strategy that attempts to address the needs brought forth through design. This design process is a collaborative effort among the researchers and users. Knowledge gained in the previous phases is used to generate responses to be vetted and tested. Prototyping is a method of testing responses in their early development in order to identify flaws and develop new investigation. It's a "thinking by doing" exercise where communication is more fluid in action (The EBD Approach Concepts and Case Studies Book, pp. 29-33).

\section{EBD Implementation}

After analyzing oral survey outcomes in phase III, investigators concluded that most themes and touchpoints from the previous phases were sensory- 
based. Further investigation included a review of literature exploring the relationship between design and sensory-based experiences, in addition to conducting additional focus groups with clinical staff and patients to validate Phase II results.

\section{Literature Review}

Review of sensory-based research informed that healing is a human experience (Sternberg, 2009). Virtually every human experience, including healing, requires our sensory motor systems (Thelen \& Smith, 2000). Kolb and Whishaw (2003) stated that:

A sensory system is a part of the nervous system responsible for processing sensory information. A sensory system consists of sensory receptors, neural pathways, and parts of the brain involved in sensory perception. Commonly recognized sensory systems are those for vision, hearing, somatic sensation (touch), taste and olfaction (smell). In short, senses are transducers from the physical world to the realm of the mind. (p. 4).

The built environment is a sensory system and sensory systems are proven to have an impact on well-being (Kolb \& Whishaw, 2003). A wide variety of sensory parameters have been shown to negatively and positively affect patient behavior and outcomes (Shabha, 2006; Wysocki, 1996; Redwine et al., 2000; Dube et al., 2008; Evans, 2001). In his review of environmental aesthetics, Antaov (1998) noted how elements in the environment can precipitate feelings of relaxation, peace, excitement and distress. Finally, the built environment impacts users' state-of-mind, which can also impact stress levels and immune system responses (Codinhoto et al., 2009).

Based on the data collected from focus groups, oral surveys and literature reviews, investigators hypothesized that the EMU patient room environment, through sensory motor system elements - vision, sounds, smells, touch and taste - has the potential to influence activity in the brain. This, in turn, may influence stress levels and healing, both of which have been found to influence pre and post seizure activity. Thus, researchers hypothesized that the patient room environment can be manipulated in ways that assist in instigating seizure and help post seizure recovery. In order to test the hypothesis, a flexible EMU patient room, capable of delivering multiple sensory motor system interventions, must be designed and tested.

\section{Design Hypothesis}

The design hypothesis focuses on the selection of design-specific elements that will intensify the sensory experience, whether to instigate a seizure or assist in recovery. Investigators reviewed best practices for the design of patient rooms from multiple case studies. They then looked for parallels in the EMU survey results and proposed applicable best practices.
The first recommendation involved the spatial layout of the patient room. Survey results indicated the need for involving family members in the patient room experience. A Clemson University report analyzing the organization of patient rooms, identified three functional zoning patterns that allow for greater family member involvement in the healing process while enabling staff to provide more efficient care and giving patients a higher sense of autonomy. A staff zone is located closer to the entryway for easy access with minimal disruption. A patient control zone is centralized in the room to balance interaction with family and care providers and family zone is designed on the peripheral of the patient zone for proximity and privacy (Clemson School of Architecture, 2003). The researchers adopted this room layout as a platform for the EMU patient room.

EMU data also suggested that patients responded to various types of visual cues. Best practices from a Patient Room 2020 study recommends the incorporation of a media wall to support virtual communication among patients, staff and family members as well as connect to audio, visual and information technology. This technology should be delivered using a concealed headwall in order to minimize physical and visual clutter (Battisto \& Ruthven, 2010). Additionally, in their "A Guide to Evidencebased Art " Kathy Hathorn and Upali Nanda (2008) emphasize the benefit of images of nature in patient rooms because, through the theory of positive distraction, it reduces the perception of pain and improves the overall healing process. To implement the visual elements in the EMU, we propose Smartboard technology. The Smartboard is a platform to upload still imagery and videos. It also supports telecom and video monitoring so the patients can stay connected to EMU staff, as well as family and friends off-site. (See image 3)

The research data supported the theories that variables of light and color influence the patient experience. A growing body of evidence suggests that light and color controls psycho-physiological responses of patients (Edelstein et all, 2008). More specifically, they impact stress levels and support recovery post procedures. Investigators proposed hybrid lighting and color applications because lighting requirements in patient rooms differ based on stakeholder needs (Bildings.com, 2009). Strobe lights and intense color lighting are also known to instigate seizure, while calm color and soft lighting support recovery. A report titled "Bringing Lighting to Healthcare" by Lightcontrol, suggests that indirect lighting supports the patient recover process. Therefore, investigators propose using reflective pigments that react to light for the wall color and colored cove lighting. The paint with reflective pigment reacts to light reflection. It is used with cove lighting to allow for easy transition of wall color to cover the full color spectrum. Based on the patient's preference, it can induce seizure activity and assist in post episode recovery by simulating a personalized recovery atmosphere at the patient's control. It can also mimic the strobe light effect on a large scale. (see images 4 and 5). 
EMU survey results also indicated that patients respond conversely and adversely to audio effects. In fact, music and noise are known to impact levels of stress, anxiety and depression in hospital environments (Richards et all, 2007). Royal Children's Hospital in Sidney, Australia implemented audio technology to provide classical music in all patient rooms. They found that the practice calms and relaxes patients significantly. The researchers propose the incorporation of patient-

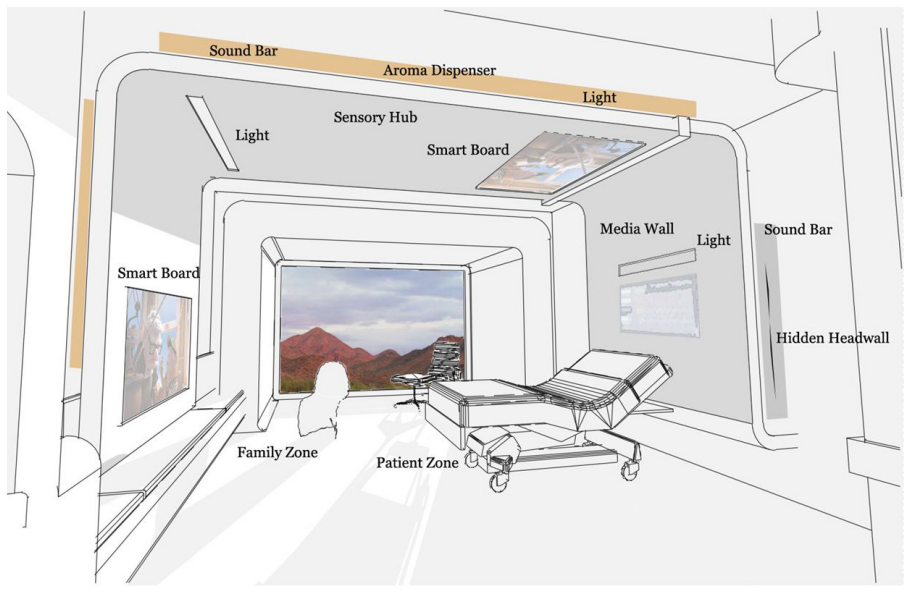

Image 3: Proposed patient room design with sensory-based hub functions

controlled surround sound to transmit music, calming sounds and white noise to aid in recovery. Seizure-inducing audio pollution like traffic noise can be transmitted as well (see image 3).

Lastly, the EMU survey data shows that patients react both positively and negatively to various types of smells. The documentary titled "The Science of Healing" by Esther Sternberg, uncovers how familiar smells can aid the healing process (Cohen \& Cohen, 2010). Based on her findings, the researchers propose the addition of an aroma induction unit to provide a variety scents, both comforting and aggravating, per the patient's preference. For taste we propose a customized eating plan consisting of a dietary program to both instigate seizure and facilitate recovery. The diets will be developed based on the patient's nutritional requirements and food preferences as they relate to causing seizures and supporting the patient post activity.

All of the above design recommendations are intended to intensify the patient sensory experience. For the purpose of this paper, the sensorybased design recommendations are referred to as the "sensory hub."

Once the tools are in place to deliver the sensory motor system interventions, EMU staff will need to identify which interventions apply to each individual patient. The researchers recommend an operational change by implementing a pre-admission interview to identify the

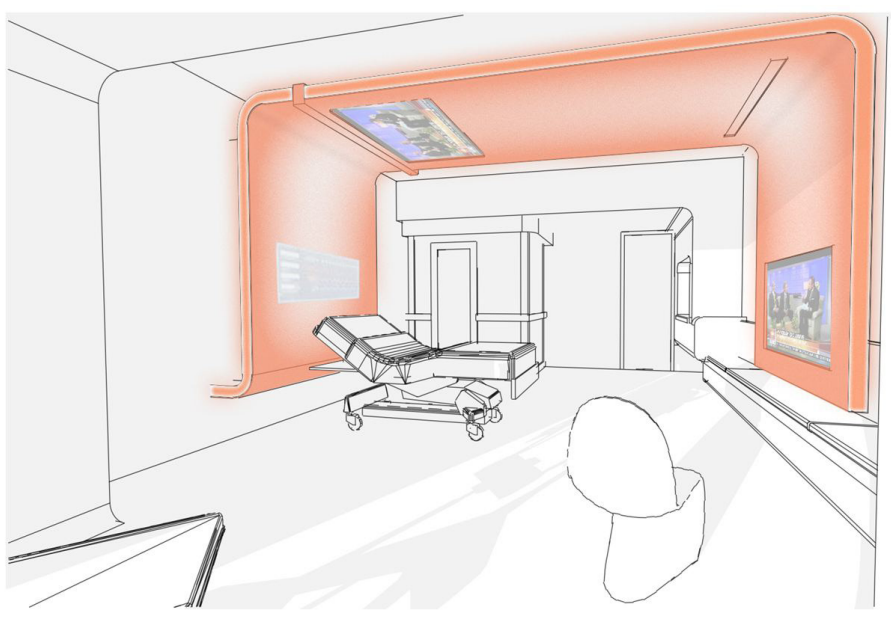

Image 4: Proposed EMU showing strobe light effect. Patients or staff can manipulate the color of the reflected light.

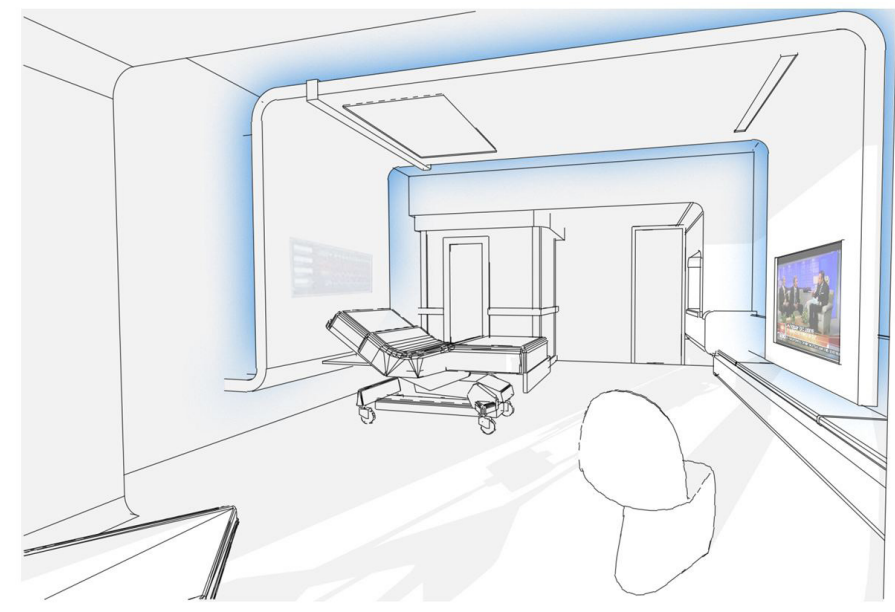

Image 5: Proposed EMU showing patient-controlled cove lighting

patients' unique triggers, recovery solutions and general preferences (see image 6). The information obtained in the interview will be used to program a unique patient room experience. We recommend that the EMU develop a comprehensive database of resources, including music, sounds, images, videos, scents, colors, lighting and menu options, to use for the interventions. The database will include both positive and negative elements in that they will be used to instigate seizures and support recovery. Staff can also recommend that patients bring in personal belongings, such as pictures, videos, books, music, audio recordings and perfume, to help program their intervention. These can be either digital or physical materials. 


\section{EBD Phase IV - Measure the Improvement}

Methodology Definition

The final phase of the EBD approach is to evaluate and test the final design solutions through qualitative and quantitative research. It is important to not only confirm the success of these solutions but to also look for continued improvement (The EBD Approach Guides and Tools Book, pp. 38-39).

\section{EMU Implementation}

For the purpose of this investigation into the design of EMU patient rooms, this phase was divided into two parts. First, we presented the proposed design solutions to multiple users, including EMU staff and The Mayo Clinic Design Team, in order to validate the recommendations and gather feedback for potential changes. The feedback was affirmative and provided minimal modifications, which we used to inform the proposed design of the EMU patient room. The second part, yet to occur, will be to build a prototype room, based on the proposed design, in order to test our hypothesis and measure the outcomes. Ultimately, we want to measure whether our proposed EMU patient room will trigger activity sooner and more frequently, as well as enhance recovery process.

\section{Next Steps: - Where Do We Go from Here?}

As mention before, a growing body of research suggests that the built environment impacts stress levels through the sensory motor system. Stress levels can control seizure activities. Thus, the researchers hypothesized that the built environment of the EMU can influence patient seizure activities. As mentioned before, this study was conducted in an environment that was design as a Medical Surgical unit, not as an Epilepsy Monitoring Unit. For future EMU designs, it is important to cultivate sensory-based design elements, including light, color, aromas, natural views, and music, within the built environment. Additionally, it is imperative for the environment to adapt to the individual patient's personal and clinical needs. Our next step in this research is to apply for another grant to finance the construction of the prototype room. ASU's research team is in the process of coordinating with the Mayo Clinic research team to investigate in potential grant proposals.

\section{References:}

Austin, J. K., Shafer, P. O., \& Deering, J. B. (2002). Epilepsy familiarity, knowledge, and perceptions of stigma: report from a survey of adolescents in the general population. Epilepsy \& Behavior, 3(4), 368375. doi: 10.1016/S1525-5050(02)00042-2.

Alcaraz, M. (2009). Lighting the Way to Good Healthcare Design. Buildings.com. Retrieved from: http://www.buildings.com/ArticleDetails/ tabid/3334/ArticlelD/8804/Default.aspx\#top

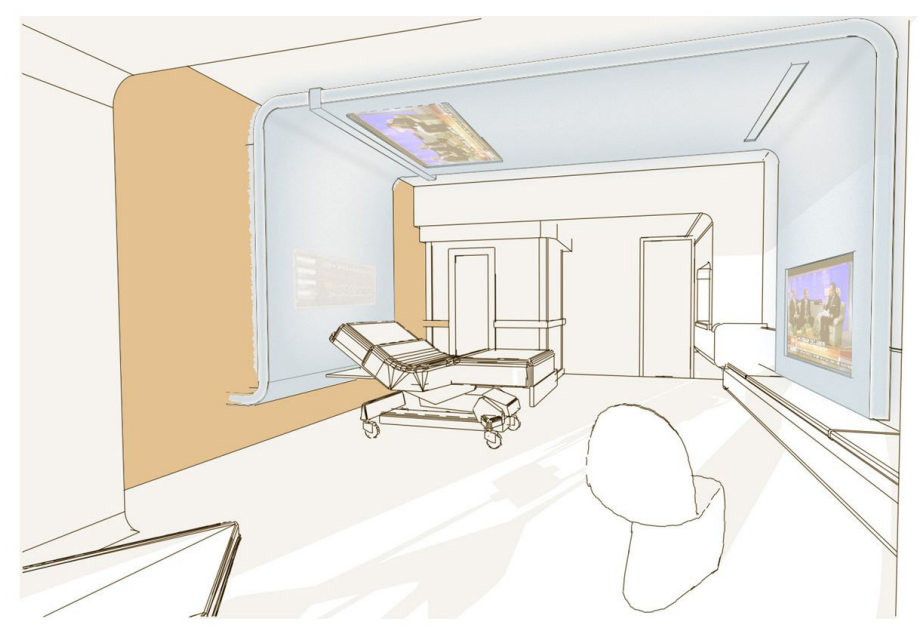

Image 6: Proposed EMU showing patient-controlled sensory hub. Patients are able to choose back wall and sensory hub reflected colors.

Antaov, Anli. (1998). Environmental aesthetics. Journal of Planning Literature 13(2), 239-257.

Baruchin, A. (February 20, 2007). Battling epilepsy and its stigma. The New York Times. Retrieved from http://www.nytimes.com/2007/02/20/ health/20epil.html.

Baker GA, Jacoby A, Buck D, et al. (1997). "Quality in life in people with epilepsy: a European study". Epilepsia 38 (3): 353-362. doi:10.1111/j.1528-1157.1997.tb01128.x. PMID 9070599.

Battisto,D. \& Ruthven, D. (2010). Patient Room / / 20 20. Retrieved from: http://media.clemson.edu/caah/architecture/faculty/posters/battisto.pdf

Baker, G. a, Spector, S., McGrath, Y., \& Soteriou, H. (2005). Impact of epilepsy in adolescence: a UK controlled study. Epilepsy \& behavior : E\&B, 6(4), 556-62. doi: 10.1016/j.yebeh.2005.03.011.

Clemson School of Architecture, (2003). Patient Room Prototype. Retrieved from: http://www.clemson.edu/caah/architecture/graduatestudents/docs/Patient-Room-Prototype-2003.pdf

Cohen, M. \& Cohen, R. (Producer \& Director). (2010). The Science of Healing [Film]. Fogelsville: Resolutionpictures.

Dube, A., Barth, M., Cutshall, S., Olson, S., Sulia, S., (2008). Environmental noise sources and interventions to minimize them: A tale of 2 hospitals. Journal of Nursing Care Quality, 23(3), 216-224. p.216. 
Evans, D. (2001). Music as an intervention for hospital patients: A systematic review. The Johanna Briggs Institute for Evidence Based Nursing and Midwifery, Adelaide.

Edelstein, E., Doctors, S., Brandt, R., Denton, B., Cranz, G., Mangel, R., Martin, M., \& Chong, G., 2008). The Effects of Colour and Light on Health: Trans-disciplinary Research Results. International Academy for Design and Health. Retrieved from: http://www.designandhealth.com/ uploaded/documents/Publications/Papers/Latrobe-Fellowship-whdapril08.pdf

Epilepsy Foundation. (N.D.) Epilepsy and seizure statistics. Epilepsy Foundation. Retrieved from: http://www.epilepsyfoundation.org/about/ statistics.cfm.

Hathorn, K. \& Nanda, U. (2008). A Guide to Evidence-based Art. The Center of Health Design. Retrieved from: http://www.healthdesign.org/ sites/default/files/Hathorn_Nanda_Mar08.pdf

Ham, C., Ellins, J., \& Parker, H. (2009). The theory and practice of markets in health care. Journal of health services research \& policy, 14(4), 193-4. doi: 10.1258/jhsrp.2009.009048.

Kolb, B. \& Whishaw, I. (2003). Fundamentals of Human Neuropsychology. New York, New York: Worth Publishers.

NHS (2009). The EBD Approach.. Adrisge, West Midlands, Institute for Innovation and Improvement.

Richards, T., Johnson, J., Sparke, A., \& Emerson, H. (2007). The Effect of Music Therapy on Patients' Perception and Manifestation of Pain, Anxiety, And Patient Satisfaction. Medsurg Nursing. Retrieved from: http://www.medsurgnursing.net/ceonline/2009/article020715.pdf

Shabha, G., (2006). An assessment of the impact of the sensory environment on individuals' behavior in special needs schools. Facilities, $24(1 / 2) ; 31-42$

Sternberg, S. (2009). Healing spaces: The science of place and wellbeing. Cambridge, MA: The Belknap Press of Harvard University Press.

Ulrich, Roger et al. (2008). A review of the research literature on evidence based healthcare design. Health Environments Research and Design Journal, 1(3).

The Centers for Disease Control. (2010). Resources, Retrieved from: http://www.cdc.gov/epilepsy/resources.htm

The Institute for Innovation and Improvement, (2010). Tools, Retrieved from http://www.institute.nhs.uk/quality_and_service_improvement_ tools/quality_and_service_improvement_tools/fundamentals_for_ quality_improvement.html

Wysocki, A. B. (1996). The effect of intermittent noise exposure on wound healing. Advances in Wound Healing, 9, 35-39. 